\title{
Spatiotemporal multiplexing method for visual field of view extension in holographic displays with naked eye observation
}

\author{
G. FINKE*, M. KUJAWIŃSKA, T. KOZACKI, and W. ZAPERTY \\ Institute of Micromechanics and Photonics, Warsaw University of Technology, \\ ul. Boboli 8, Warsaw, Poland
}

\begin{abstract}
In this paper we propose a method which allows to overcome the basic functional problems in holographic displays with naked eye observation caused by delivering too small images visible in narrow viewing angles. The solution is based on combining the spatiotemporal multiplexing method with a 4 foptical system. It enables to increase an aperture of a holographic display and extend the angular visual field of view. The applicability of the modified display is evidenced by Wigner distribution analysis of holographic imaging with spatiotemporal multiplexing method and by the experiments performed at the display demonstrator.
\end{abstract}

Keywords: digital holography, holographic display, 3D imaging, visual field of view, spatiotemporal multiplexing.

\section{Introduction}

Three-dimensional visual perception is the most natural way for humans to experience the real world. No wonder that a lot of effort has been put recently to add the $3^{\text {rd }}$ dimension into imaging technology. Nowadays, the biggest commercial implementation of 3D imaging and display is mainly realized by stereoscopic, pseudostereoscopic and integral imaging techniques which are based on 2D images and their transformation by our brain $[1,2]$ In order to provide a "true$-3 \mathrm{D}$ " display, it is necessary to deliver a high quality optical replica of an object or scene that can be viewed by an observer freely from different directions. This can be achieved with holography that stands out from the other 3D imaging techniques [3] due to the ability to capture and correctly reconstruct $3 \mathrm{D}$ object wavefront $[4,5]$.

Technological developments in the area of spatial light modulators, high resolution cameras and computers with high computing powers have provided the means to transfer holographic imaging concept into a digital world. However, the biggest difficulty in this task is to provide a holographic display with sufficient spatial bandwidth product (SBP) which is required for holographic representation of a 3D object with respectable sizes. In order to solve this problem two main approaches are proposed. The first one relies on holographic reconstruction of small angular parts of an object wavefront (from a large volume 3D scenes) that actually reach observer eyes. The wavefronts are updated accor-

*e-mail: g.finke@mchtr.pw.edu.pl ding to the information provided by an eye tracking system [6]. This solution is used for a single observer or, recently, a limited number of observers systems [7]. It has already been well described and is commercially available since in this solution technological development (SLM parameters) is already sufficient. The second approach relies on creating high resolution, big aperture (with high pixel count) holographic display which provides simultaneously (in reference to human vision system inertia) total information about displayed image within a wide viewing zone for multiple observers [8]. The good example of the second approach is the "active tiling" system developed by QuinetiQ [9], however, the hardware connected with this system (based on combination of electrically and optically addressed SLM and multiple optics) is highly complicated. The second approach is also realized by means of creating larger display through combining reflective type SLMs into flat or curved panel [10-14]. The most common devices used in holographic displays are Liquid Crystal on Silicon (LCoS) spatial light modulators (SLMs) which provide the highest diffraction efficiency and reconstruction accuracy [16]. However, they have now several technological limitations and suffer from insufficient value of spatial bandwidth product, i.e., they have a large pixel size and small aperture [11]. In order to overcome these problems it is necessary to seek solutions for the SBP increase, that enlarge viewing angle and image dimensions.

The first problem is widely addressed in literature. One of the solutions is based on spatial multiplexing technique in 
which several SLMs are arranged on an arc that increase horizontal viewing angle [13-15]. Such approach is highly expensive since the viewing angle depends on the number of used modulators. Also due to the size of physical frames of SLMs, it is difficult to obtain a continuous aperture of a display. Another way to increase SBP is an implementation of temporal multiplexing [17-19] in which a number of SLMs, from which information originates, increases virtually. This is possible by modulating a direction of updated light field propagation with the frequency above eye refreshing rate $(50 \mathrm{~Hz}$ preferably). In this way a continuous row or matrix of virtual SLMs is generated [17]. However, the more virtual SLMs appear, the higher refresh rate an SLM should work with. To lessen this condition spatiotemporal multiplexing methods which utilize several physical SLMs multiplied temporally are used [8].

The second problem, namely the limited size of an image, is related with an observation method. In the desired naked eye observation method, the image size is always limited by an SLM aperture; we experience so called "key-hole" effect. This limitation cannot be changed, however several approaches are implemented to bypass it by means of other observation techniques or extension of a display size. One of the observation techniques that allows for reconstruction of images with bigger size than SLM's aperture applies an additional medium, namely asymmetric diffuser [20]. Here, on the contrary to the naked eye observation method, an image is scattered in vertical direction which allows to see more information vertically (up to the size of FOV) but the vertical parallax is lost. In horizontal direction the size of an image is still limited by an SLM size, but it contains 3D information (limited horizontal parallax) [13] which can be extended by horizontal spatial multiplexing. Another way is to use spatial multiplexing in horizontal and vertical directions and appropriately designed optical system, as shown in Ref. 21.

An alternative method, which we propose in this paper is an application of spatiotemporal multiplexing method (STM) which provides significant increase of viewing angle as described in Ref. 17 combined with a sequence of an image demagnifying and magnifying operations performed by an appropriate $4 \mathrm{f}$ optical system. The paper is organized as follows. In Sect. 2 we present the concept of the method. Next (Sect. 3), the display system is analysed by means of Wigner distribution performed in the planes of SLMs and holographic image. The methodology of experiments and the final image reconstructions obtained in the developed display system are presented in Sects. 4 and 5, respectively. The advantages of the system and perspectives of its further development are discussed in Sect. 6.

\section{The basic concept of the method}

The concept of our method is illustrated in Fig. 1. Four phase only reflective SLMs are aligned in a $2 \times 2$ array on a circle with the fill factor $\mathrm{FF}=0.5$ in both horizontal and vertical direction. A beam splitting element BS is used to create two rows of SLMs and to illuminate them with a plane wave propagating along optical axis. The SLM M is placed at the plane $\pi^{\prime}$ conjugated with a holographic image reconstruction
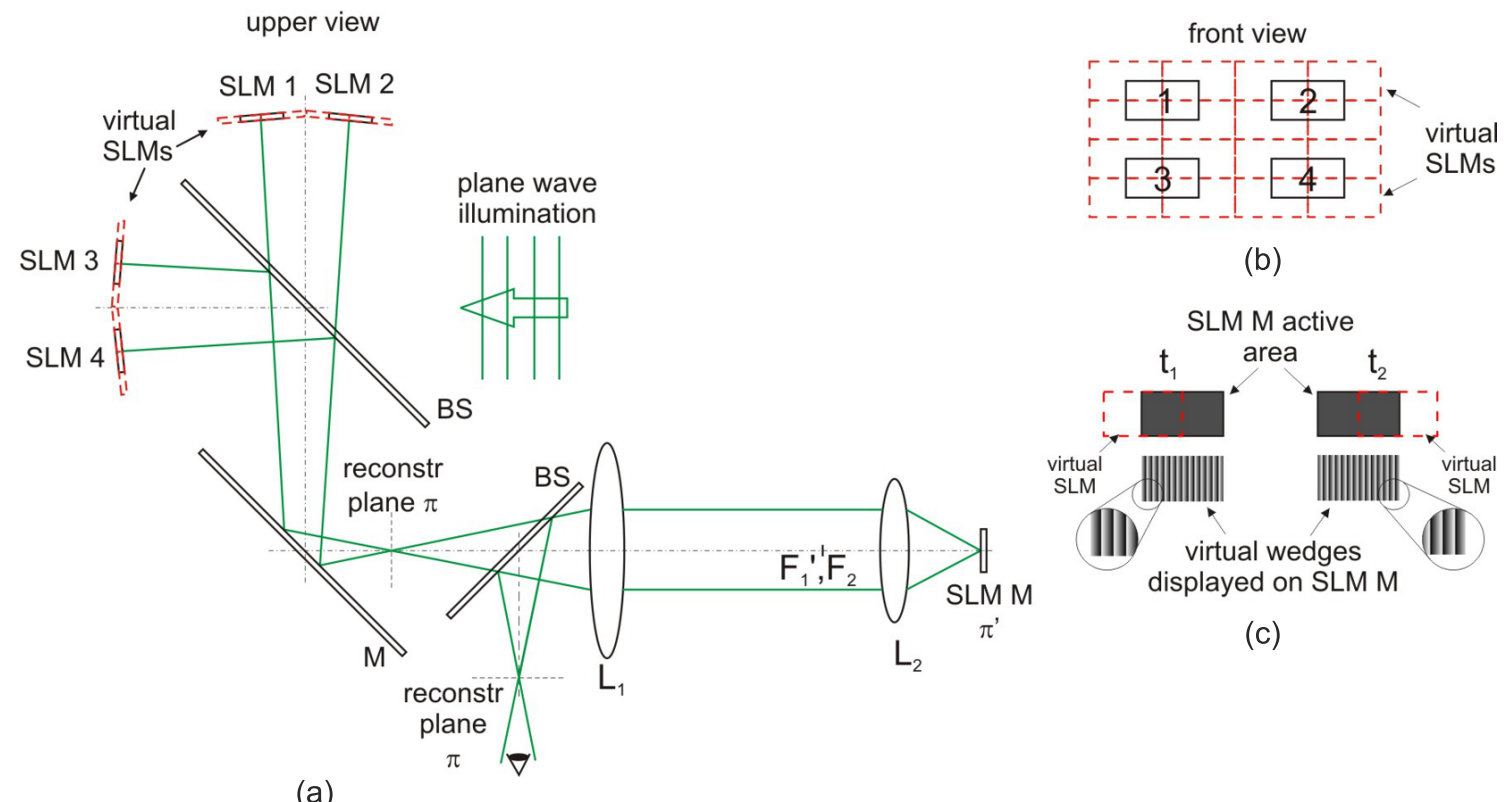

(b)

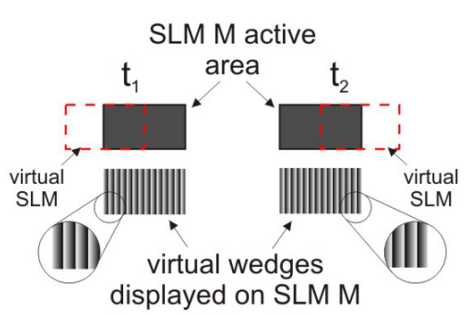

(c)

(a)

Fig. 1. The scheme of the multi SLM display with enlarged image size: (a) the scheme of full system, (b) the spatial arrangement of real and virtual SLMs in experimental setup, (c) exemplary creation of virtual wedge in time for single SLM in horizontal direction. 
plane $\pi$ through a 4 f optical system composed of lenses $L_{1}$ and $\mathrm{L}_{2}$ of different focal lengths. The proper choice of the focal lengths provides the transverse magnification $\mathrm{M}_{\mathrm{t}}<1$, and angular magnification $\mathrm{M}_{\alpha}=1 / \mathrm{M}_{\mathrm{t}}>1$. The final image enlargement is obtained in two steps: in the first one the reconstructed image is decreased to the size matched to SLM $\mathrm{M}$ dimensions, in the second step this image is back propagated through a $4 \mathrm{f}$ system and its size is increased to the size of an image observed by a naked eye.

The role of SLM M in the setup is to redirect an incident beam originating from $\mathrm{i}$-th SLM (here $\mathrm{i}=1, \ldots 4$, but it can be increased) at a desired angle $\alpha_{x / y}$. It is realized by addressing SLM M with linear phase $\bmod (2 \pi)$ (virtual wedge) given by $\alpha_{x / y}=\pi \lambda A_{x / y}$, where $\pm A_{x / y}$ is the spatial frequency of the phase fringes in direction $x$ or $y$. The example of virtual SLM positions for the case of a single SLM is shown in Fig. 2 as the red, dashed-line rectangles.

The principle of work of a 2D matrix of virtual modulators [Fig. 1(b)] is the same, however, in order to obtain clear image without flickering faster SLMs are required. To compose a full object frame (4 positions of virtual SLMs) the time shorter than the inertia of human's eye is necessary. Nevertheless, in a final result the increased number of virtual SLMs will result in increase of the visual field of view (amount of information obtained by an observer) and, therefore, it will enhance visual perception conditions.
The selection of magnifications given by the $4 \mathrm{f}$ system plays a key role in the display and depends on several parameters such as: angular separation of adjacent SLMs, SLM M pixel size and size of reconstructed image. The biggest limitations come from SLM's features, i.e., its aperture and pixel size. Due to SLM structure the maximum angle at which it can reflect an incident beam equals $\alpha_{S L M M}= \pm \lambda / 2 \Delta_{x}$ (where $\lambda$-wave length, $\Delta_{x}$ - pixel pitch). The angle after back propagation of an object beam through the $4 \mathrm{f}$ system is decreased $M_{\alpha}$ times. Therefore, assuming the maximum shift of SLM by half of its size, the following relation can be written

$$
\frac{\alpha_{S L M M}}{M_{\alpha}}=\frac{B_{x}}{2 z_{r}},
$$

where $B_{x}$ is the SLM size, $z_{r}$ is the reconstruction distance.

The relation given by Eq.(1) shows that with an increase of the angular magnification $M_{\alpha}$ (decrease of transverse one) the reconstruction distance $z_{r}$ has to increase which translates into smaller viewing angles. On the other hand, such situation would be advantageous for bigger images since they could be appropriately decreased to a SLM's size. Therefore, a certain compromise has to be chosen based on the setup parameters and viewer's expectations. The relation in Eq.(1) could become more loose if SLM M pixel size decreased. Then $\alpha_{S L M M}$ would increase, hence bigger $M_{\alpha}$ could be used without ne-

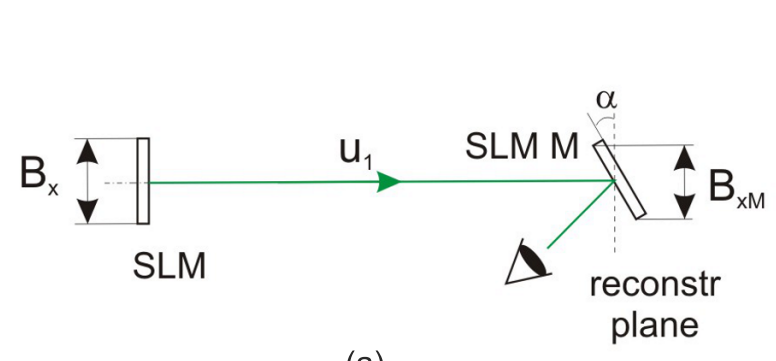

(a)

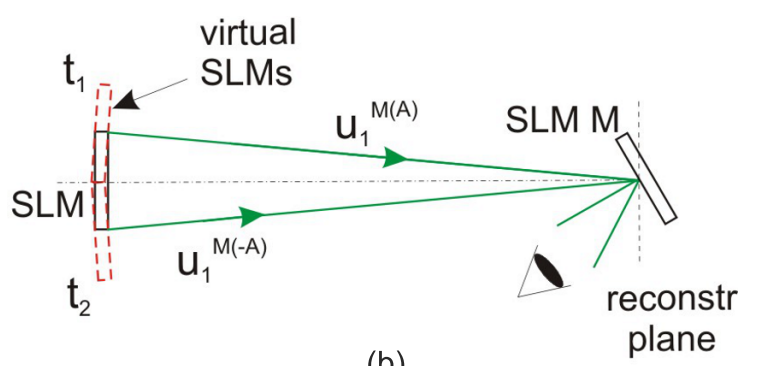

(b)

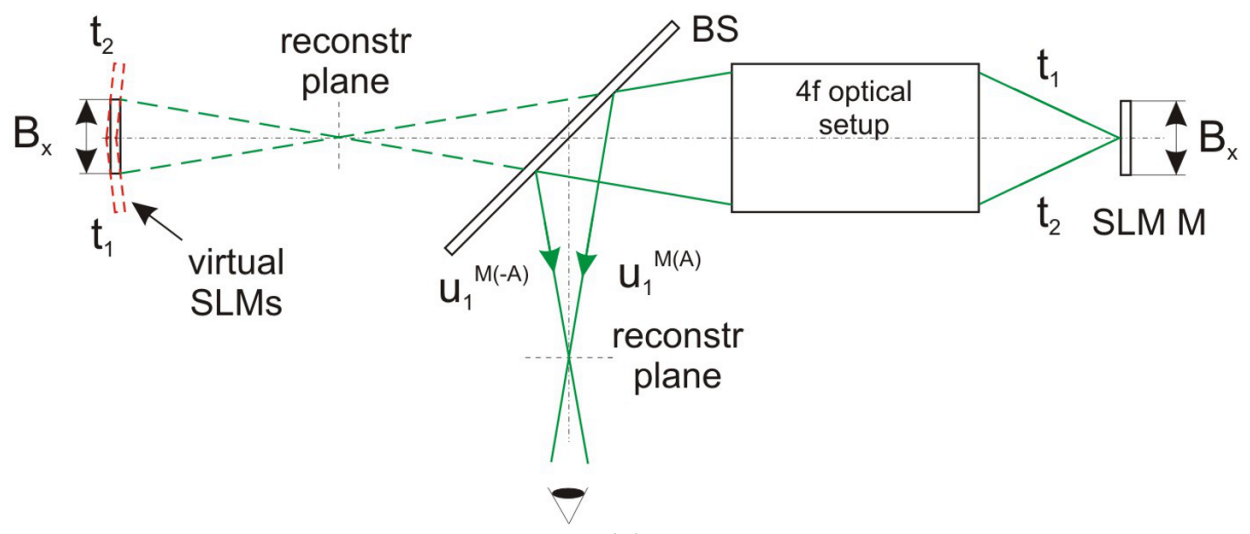

(c)

Fig. 2. The schemes of the setup with additional SLM M illustrating the working concept of the display: (a) SLM M switched off, (b) SLM M switched on, (c) setup with 4 f optical system. 
cessity of $z_{r}$ increase. Also bigger SLM M aperture (without pixel size increase) would be beneficial since then smaller angular magnifications could be used.

\section{Wigner distribution analyses of holographic imaging with STM method}

The analyses of holographic imaging with the described STM method are carried out by means of Wigner distribution as it gives possibility to illustrate local information about reconstructed signals in both frequency and space [22]. In order to show general character of the signals, they are analysed with no specified values such as reconstruction distance $\left(z_{r}\right)$, display size $\left(B_{x}\right)$, pixel size $\left(\Delta_{x}\right)$, etc. SLMs are illuminated in the same way as shown in Fig. 1. Therefore, (to keep the schemes simple) the SLMs illumination is not shown in Fig. 2.

At first the analysis of the setup is performed for a single SLM, without optics and with SLM M turned off in the configuration shown in Fig. 2(a). The SLM is illuminated with a plane wave, the hologram is reconstructed at the distance $z_{r}$ in which the additional SLM M is placed in order to redirect the incident field $u_{1}$. The Wigner distribution of an SLM M and holographic signal in the reconstruction plane for this case is presented in Fig. 3(a.) The green area represents the amount of information which observer receives during naked eye observation. The area is limited in horizontal direction by the size of SLM M and since SLM is tilted at an angle $\alpha$, it equals $B_{x M}=B_{x} \cos \alpha$. Fig. 3(a) shows that in general during image reconstruction with single SLM a lot of SBP is lost. In order to optimize the usage of SBP, multiplexing techniques are implemented through activating SLM M. The case with SLM M turned on is illustrated in Fig. 2(b) and Fig. 3(b). By displaying at SLM M linear phase of a properly chosen frequency the incident field $u_{1}$ is redirected at a desired angle. In the result a copy $u_{1}^{M}$ originating from different location is being created. If SLM M is modulated in time interchangeably with a plane wave with inclination represented by the spatial frequencies $\pm A$, a set of virtual SLMs will appear determining new places of signals $u_{1}^{M( \pm A)}$ origin as illustrated in Fig. 2(b). The spatial frequency $( \pm \mathrm{A})$ is selected depending on display's features, i.e., reconstruction distance $z_{r}$, modulator's size $B_{x}$ and fill factor FF and translates into reflected plane waves with frequencies $f^{M( \pm A)}= \pm B F F /\left(\lambda z_{r}\right)\left[\mathrm{mm}^{-1}\right]$. In Fig. 3 and Fig. 2(b) WD of real and virtual SLMs are presented. The received amount of information (green area) is still limited by $B_{x M}$, however, a certain profit in the frequency domain can be obtained which translates into an increase of a viewing angle. The conclusion is that, in order to observe images bigger than the size of SLM, its dimensions need to be increased. In Fig. 3(b) such solution is presented, where reconstruction plane $\pi$ is conjugated with SLM M thanks to adding $4 \mathrm{f}$ opti- cal setup. The role of SLM M remains the same as in the previously described case, i.e., change of incident light direction. The difference is that due to implementing the optical setup the transverse and angular magnification are introduced $\left(M_{t}<1\right.$ and $\left.M_{\alpha}=1 / M_{t}\right)$. In Fig. 3(c) WD of SLM M and incident signal $u_{1}$ are presented. Maximum value of $u_{1}$ shift in time which SLM M can provide is illustrated with green solid lines $\left[u_{1} \mathrm{M}(\mathrm{A}), u_{1} \mathrm{M}(\mathrm{A})\right]$ and depends on pixel size of SLM M. If a continuous image is to be obtained [Fig. 2(d)] the necessary frequencies $f^{M( \pm A)}$, which are increased by angular magnification $M_{\alpha}$ have to be achievable by SLM M. This means that modulation applied on SLM M $( \pm \mathrm{A})$ according to Niquist theorem, due to limited modulator's pixel size, need to fit $M_{\alpha}|A|<1 / 2 \Delta_{x}$ condition. Due to magnification provided by the $4 \mathrm{f}$ system, resolution (RES) of an image changes. The situation is similar to the case when hologram reconstruction distance decreases and can be described by the following relations

$$
\begin{aligned}
\mathrm{RES}_{1} & =\mathrm{RES} / \mathrm{M}_{\mathrm{t}} \\
\mathrm{z}_{1} & =\mathrm{z}_{\mathrm{r}} \mathrm{M}_{\mathrm{t}} .
\end{aligned}
$$

The big advantage of the presented solution over the one without optical system is that the size of an observed image depends on a $4 \mathrm{f}$ setup magnification and can vary depending on an observer's needs. It is scalable and the addition of more SLMs in horizontal or vertical direction brings no conceptual problems, however, it may cause significant increase of the size and cost of the setup.

\section{Experimental setup analyses}

The experimental setup (Fig. 1) was composed of 4 phase only SLMs (HEO 1080P [23]) aligned on a circle with fill factor $\mathrm{FF}=0.5$ in both horizontal and vertical direction. A beam splitting element BS was used to create second row of SLMs. Composition of a $4 \mathrm{f}$ setup, which conjugates reconstruction plane with SLM M, depends on the final expected effect. In order to obtain big FOV the reconstruction distance $z_{r}$ needs to be big. We have chosen to reconstruct hologram at $z_{r}=700 \mathrm{~mm}$, for which FOV $=46.5 \mathrm{~mm}$. Such reconstruction distance together with a distance between two adjacent SLMs in horizontal direction resulted in their angular separation of $\pm 0.63^{\circ}$. Considering illumination with $\lambda=$ $532 \mathrm{~nm}$ and maximum diffraction angle of an SLM for this $\alpha_{S L M M}= \pm 1.9^{\circ}$, the $4 \mathrm{f}$ setup magnification should not be bigger than $M_{t}=0.63 / 1.9=0.33$. This is why our $4 \mathrm{f}$ setup was built from lenses with focal lengths $f_{1}=300$ and $f_{2}=$ $100 \mathrm{~mm}$. In the vertical direction the shift between two adjacent SLMs was smaller, hence restriction for optical setup magnification was weaker. Finally, considering SLM's dimensions and shift of virtual modulators by half of their dimensions in both directions $\mathrm{X}$ and $\mathrm{Y}$, we needed 4 subframes 


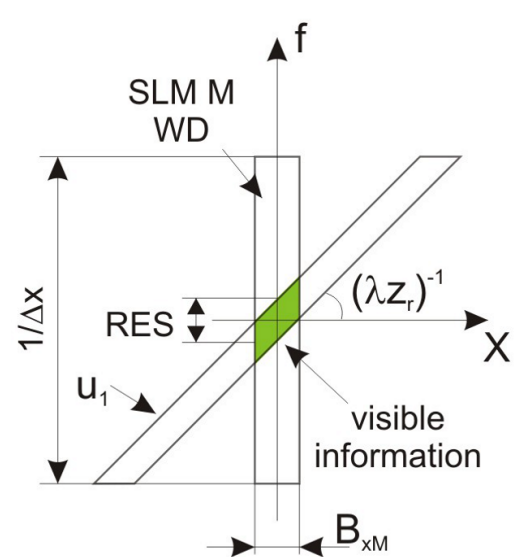

(a)

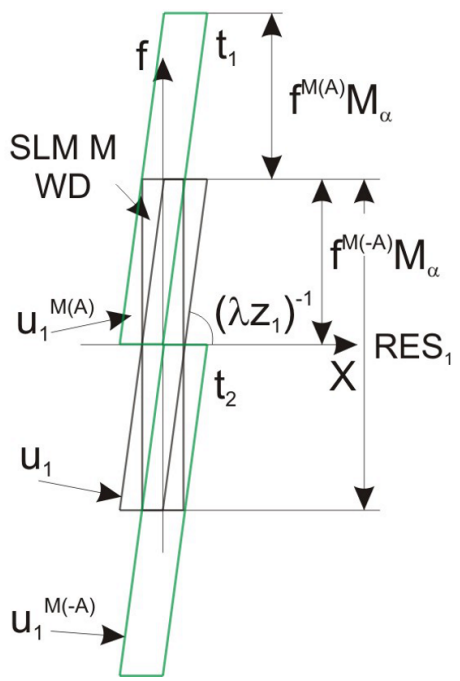

(c)

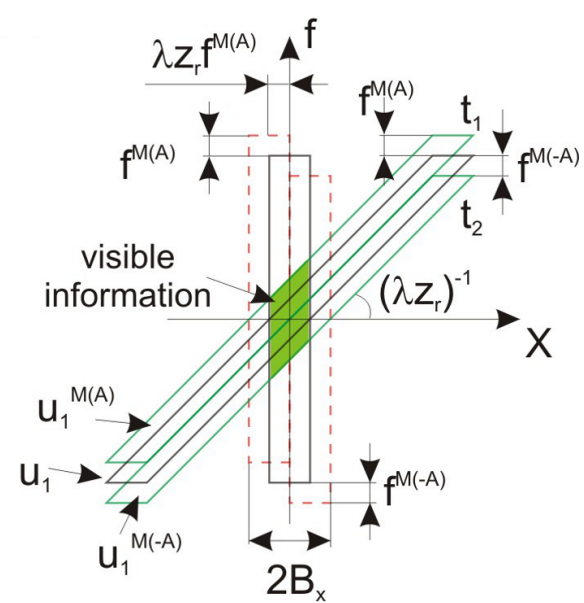

(b)

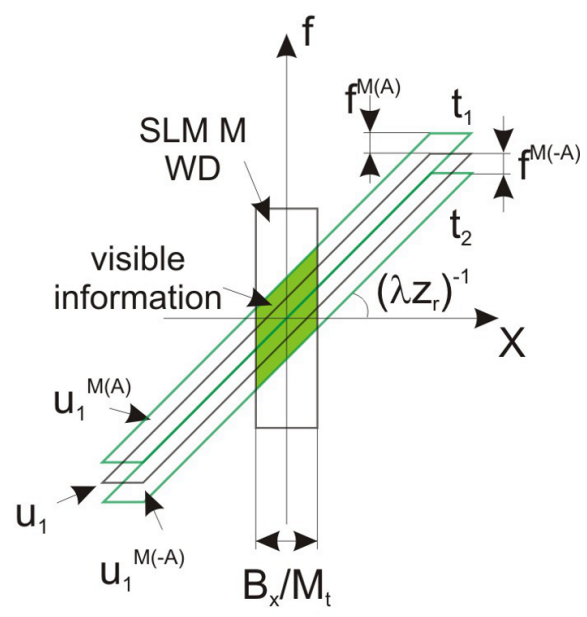

(d)

Fig. 3. Wigner distribution of holographic signal representing different setup configurations: (a) SLM M switched off, (b) SLM M switched on, and setup with $4 \mathrm{f}$ optical system analyzed at: (c) SLM M plane, (d) reconstruction plane.

delivered sequentially in time with spatial frequencies $f^{M}=$ $\left[ \pm 62 \mathrm{~mm}^{-1}, \pm 35 \mathrm{~mm}^{-1}\right]$ which will shift virtual modulators along diagonals of the real ones.

Finally our system provided the following features, when compared with a single SLM reconstruction:

- increase a viewing angle, while keeping continuity of the reconstructed image,

- increase of the visual field of view,

- increase of the image observed with a naked eye.

In order to obtain a reliable and continuous image reconstruction the spatiotemporal multiplexing is implemented through synchronizing SLM M with the set of a 4 real SLMs at which the sequential holograms are displayed. The fast hologram display is performed through a direct GPU addressing with the prepared holograms. In theory maximum fre- quency with which the system delivers final image (consisting of 4 subframes) should equal $15 \mathrm{~Hz}$, as maximum frame refreshing rate of an SLMs is $60 \mathrm{~Hz}$. In practice, due to hardware limitations, the final image has been delivered with the frequency $12.5 \mathrm{~Hz}$.

Another advantage of combining temporal and spatial multiplexing is the increase of display's visual field of view (VFOV). VFOV defines the size of an area observed by human's eye and can be calculated from the following equation [24]

$$
\operatorname{VFOV} V_{x, y}=\frac{B_{x, y} z_{o}}{z_{o}+z_{r}}
$$

where $B_{x, y}=N_{(x, y)} \Delta_{(x, y)}$. 
VFOV depends on the number of pixels $N_{(x, y)}$, their size $\Delta_{(x, y)}$, as well as observation and reconstruction distances $z_{o}$, $z_{r}$. This means that for fixed reconstruction conditions and a single modulator case, in order to increase VFOV an observer has to move away from a reconstructed image (increase of $z_{0}$ ). Thanks to application of the STM method, we are able to "change reconstruction conditions" by increasing of the display size (here $4 \mathrm{~B}_{\mathrm{x}}$ ), thus observation distance can be reduced and, moreover, the reconstructed image becomes bigger.

However, due to a certain limitations of the optical system, it is necessary to properly choose its parameters in relation to the other features of the display. Basically, there are two factors that need to be looked into, in order to estimate the final acceptable size of an image:

1. SLM M size and transverse magnification $\mathrm{M}_{\mathrm{t}}$. Image demagnified by $4 \mathrm{f}$ setup must fit SLM M dimensions $\left(N_{(x, y)} \Delta_{(x, y)}\right)$, therefore its original size cannot be bigger than $N_{(x, y)} \Delta_{(x, y)} / M_{t}[\mathrm{~mm}]$.

2. Principal ray propagation in $4 \mathrm{f}$ setup and display configuration. It has to be analyzed by means of geometric optics in order to properly select the dimensions of both lenses or acceptable image size.

In our setup we have chosen to use lenses with the following parameters: $\mathrm{f}_{1}=300 \mathrm{~mm}, \phi_{1}=75 \mathrm{~mm}\left(\mathrm{~L}_{1}\right)$ and $\mathrm{f}_{2}=$ $100 \mathrm{~mm}, \phi_{2}=50 \mathrm{~mm}\left(\mathrm{~L}_{2}\right)$. Taking into account the above considerations, the transverse magnification of the optical system is $M_{t}=1 / 3$, which together with SLM M dimensions $15.36 \times 8.64 \mathrm{~mm}(\mathrm{~W} \times \mathrm{H})$ limited the acceptable image size to $46.08 \times 25.92 \mathrm{~mm}(\mathrm{~W} \times \mathrm{H})$. The aperture stop $A_{s}$ is located at the second lens. Therefore, natural vignetting is given by the first lens, which results in max. principle ray angle $\alpha_{p}=1.79^{\circ}$, i.e., the possible image size $\phi_{p}=56 \mathrm{~mm}$. The experimental display, concerning virtual modulators, has the largest dimension in horizontal direction $(4 \times 15.36=61.44 \mathrm{~mm})$ which translated into image acceptable area $\phi_{d}=34 \mathrm{~mm}$. The principal ray propagation in our setup and display configuration influence on image size is illustrated in Fig. 4. At SLM plane real SLMs are represented by black rectangles, whereas virtual by red dashed lines (see also Fig. 1).

Finally, looking at the obtained values, the display with implemented STM method provided the image with the increased size, namely 3 times bigger height and 2 times bigger width of the image in comparison to a single SLM size.

\section{Experimental results}

To verify functionality of the display the experiments had been performed in two steps: reconstruction of holograms of a target 2D object and reconstruction of holograms of a real 3D object - gargoyle statue. For the target object a simple 2D image was used, whereas 3D data of gargoyle statue had been acquired by means of the fringe projection system [25],

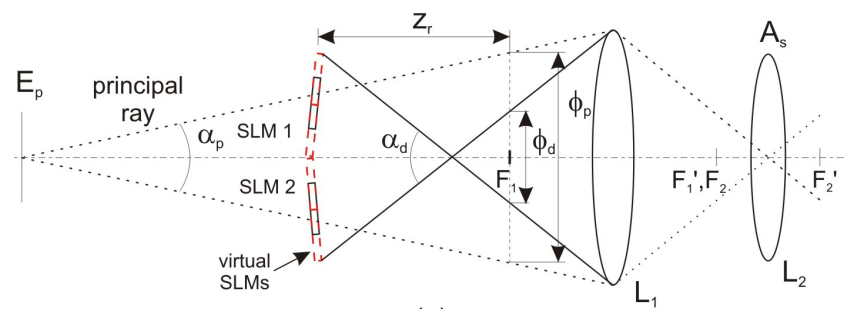

(a)

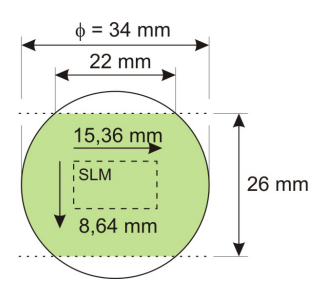

(b)

Fig. 4. The geometrical optics analysis of the display: the analysis of (a) image size based on principal ray propagation and display configuration $\left(A_{s}\right.$ - aperture stop, $E_{p}$ - entrance pupil), (b) final size of the reconstructed image area in the experimental setup (green field) with a single SLM inscribed for size comparison.

which provides cloud of points $(\mathrm{x}, \mathrm{y}, \mathrm{z})$ representation. The phase representation of both objects wavefronts at the hologram planes were generated using Gerchberg - Saxton algorithm [26]. The phase values had been displayed directly on SLMs. Such approach allowed for zero order and additional diffraction orders suppression.

At first the target object [Fig. 6(a)] which fits the area shown in Fig. 4(b) (green field), had been designed and a set of holograms corresponding to the tilt applied at SLM M were calculated. To mimic human observation a digital camera was located on axis in $z_{o}=960 \mathrm{~mm}$ distance from a reconstructed image with its diaphragm diameter set to 5 $\mathrm{mm}$ (human's pupil diameter in a dark room). The exposure time of the camera was set to $1 \mathrm{sec}$. The input power of used lased equalled app. $5 \mathrm{~mW}$, however, for naked eye observation, due to safety reasons, it had to be decreased to single $\mu \mathrm{W}$. Illumination power and camera parameters remained the same during all experiments. For better visualization of our method, the reconstructions have been captured for four different modulation states: SLM M turned off, i.e., $f^{M}=[0$, $0]$, and SLM M turned on with: $f^{M}=\left[ \pm 62 \mathrm{~mm}^{-1}, 0\right]$ (horizontal multiplexing), $f^{M}=\left[0, \pm 35 \mathrm{~mm}^{-1}\right]$ (vertical multiplexing) and $f^{M}=\left[ \pm 62 \mathrm{~mm}^{-1}, \pm 35 \mathrm{~mm}^{-1}\right]$ (diagonal multiplexing). The shift of virtual modulators (red, dashed lines) in respect to the positions of real SLMs for each modulation state applied on SLM M in particular time moment $t_{1}$ is shown in Fig. 5, whereas the experimental results in Fig. 6 and Fig. 7. In Fig. 6(b) when SLM M is turned off the image can be seen as reconstruction of specific parts of an object throughout four "key holes", each one associated with the real SLMs. When SLM M is turned on, depending on the 


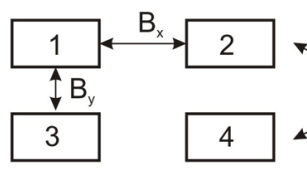

(a)

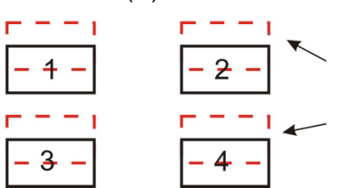

(c)
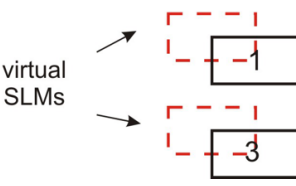

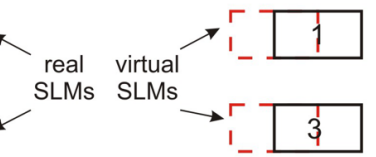

(b)
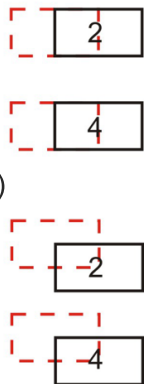

(d)
Fig. 5. Positions of virtual modulators for different modulation states applied on SLM M in moment $t_{1}$; (a) SLM M turned off, (b) horizontal shift, (c) vertical shift, (d) diagonal shift.

applied modulation direction one of the previous gaps disappears [Fig. 6(c) and 6(d)]. For best coverage of VFOV in both directions the diagonal positions of virtual modulators created by 4 temporal subframes had been implemented [Fig. 6(e)]. As stated before, an observer's position is crucial for proper amount of information receipt. This is why we have placed our camera $960 \mathrm{~mm}$ from the reconstructed image, which gives $\mathrm{VFOV}_{\mathrm{y}}=20 \mathrm{~mm}$ (for 4 virtual modulators). This result is significant in comparison to a single modulator case, where for the same observation conditions $\mathrm{VFOV}_{\mathrm{y}}=5 \mathrm{~mm}$. Utilization of the proposed technique, however, has its impact on decrease of average intensity in larger visual field of view area. Therefore, for the same exposure time value, the intensities of captured images presented in Fig. 6(b)-6(e) differ from each other - the highest Fig. 6(b), the lowest Fig. 6(e). Because of that also the noise visible in images Fig. 6(b)-6(d) is less noticeable in the reconstruction presented in Fig. 6(e). The same statements hold for the results presented in Fig. 7.

In the next step we reconstructed the hologram of the real 3D object. The reconstructed image was $15 \mathrm{~mm}$ high, i.e., only a little bit smaller than VFOV calculated for the observation distance $z_{o}=600 \mathrm{~mm}$ (Fig. 7). The presented results, as previously, show the effects of imaging in the display for the case of implementation of 4 different sets of modulation frequencies. The final image, in which observation of good quality continuous reconstruction of $3 \mathrm{D}$ object is possible, shows well the potential of the proposed method. The presented system allows for 4 times more information perception in comparison to a single modulator (for the same observation parameters).

It should be also noticed that due to relatively low frame refreshing rate of the utilized SLMs $(60 \mathrm{~Hz})$ flickering effect was noticeable (12.5 Hz/frame). This, however, is a technological problem, which should decrease or even disappear for the SLMs with higher refreshing rates. Faster SLMs will also affect higher mean intensity distribution in VFOV area, which will additionally improve quality of observed reconstructions.

\section{Conclusions}

The paper describes a holographic display based on 4 SLMs aligned on an arc and additional SLM M used for modulation of image wavefronts. In a contrary to our previous works, we had focused on providing the possibility to obtain a reconstructed image for naked eye observation with a size much

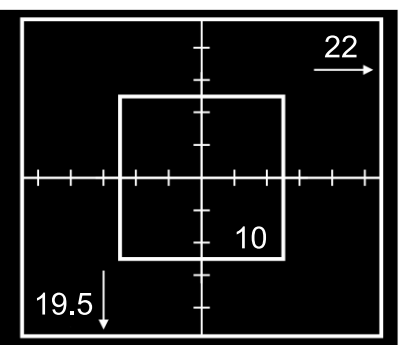

(a)

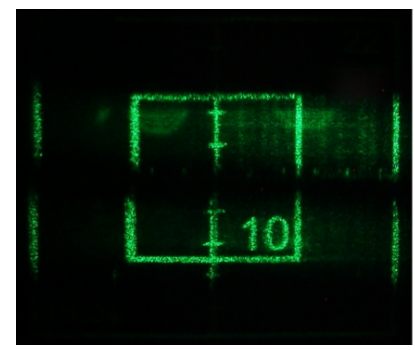

(c)

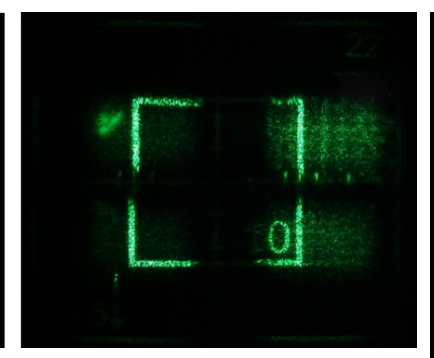

(b)

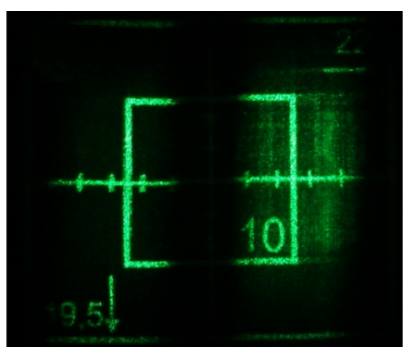

(d)

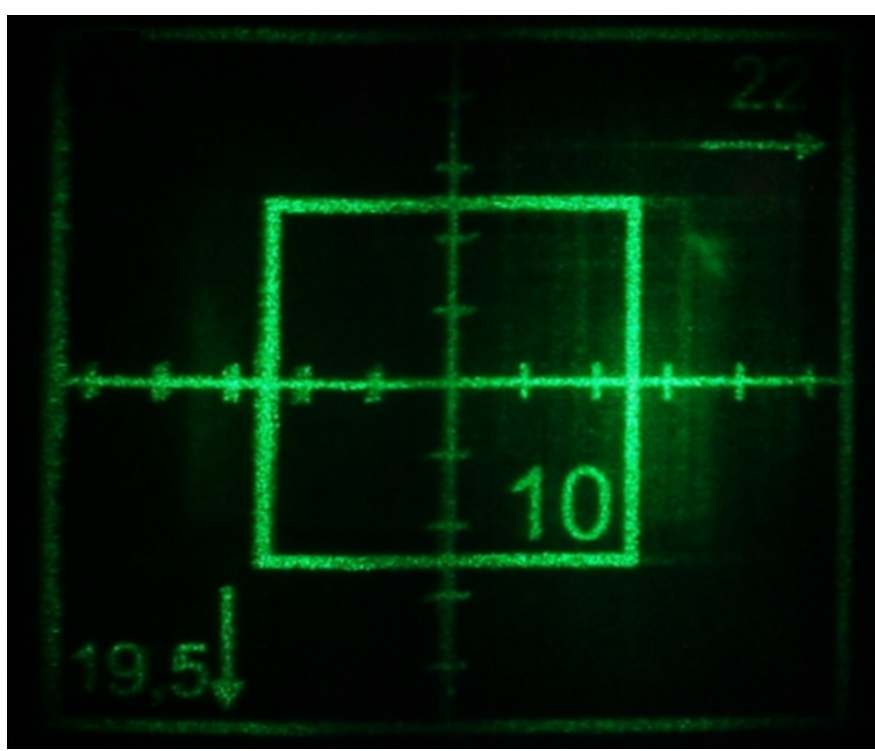

(e)

Fig. 6. 2D target: (a) the original object, and holographic reconstructions obtained with: (b) SLM M turned off, (c) horizontal multiplication, (d) vertical multiplication, (e) diagonal multiplication. 


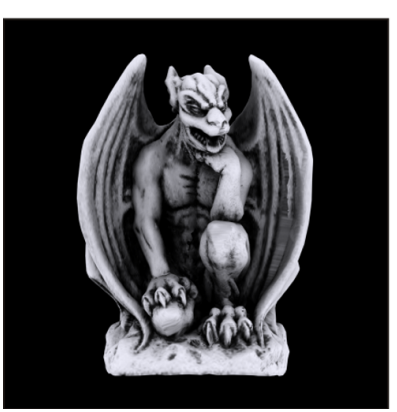

(a)

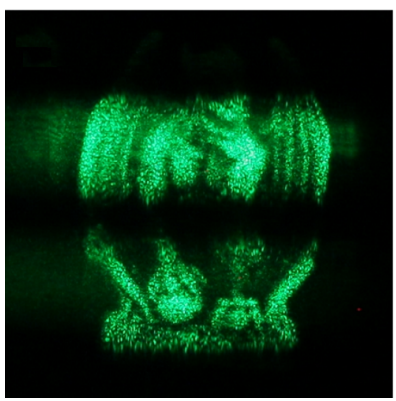

(c)

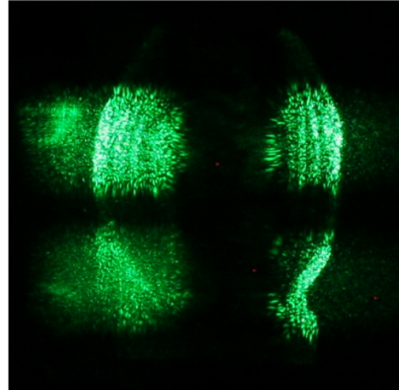

(b)

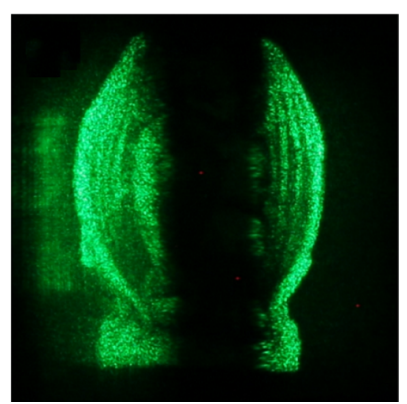

(d)

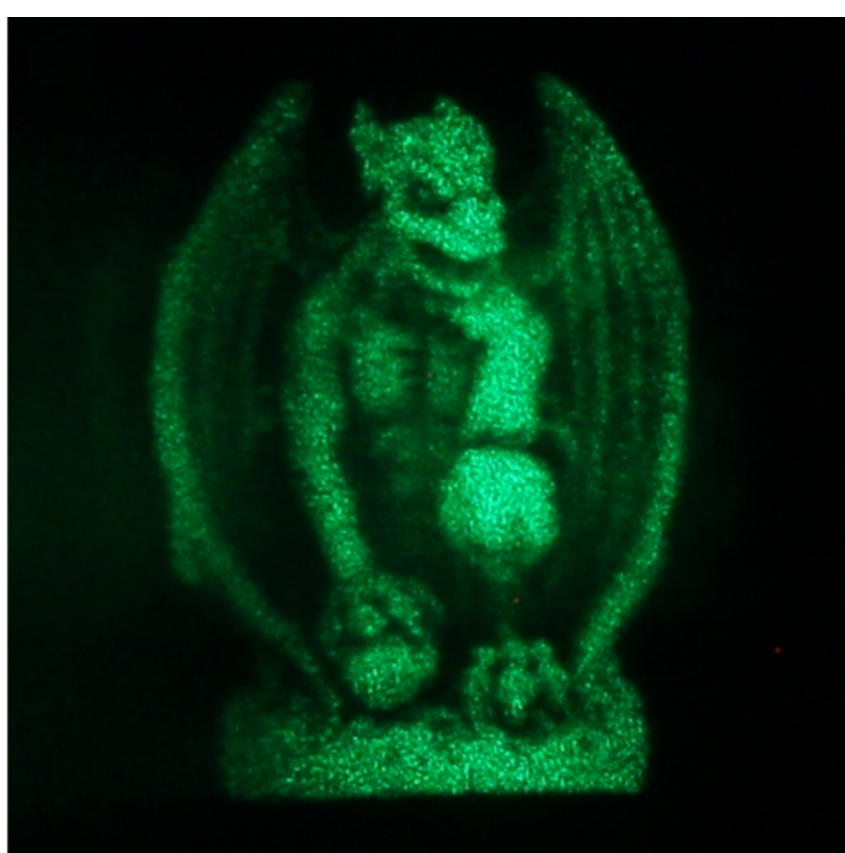

(e)

Fig. 7. Gargoyle statue: (a) the original object, and holographic reconstructions obtained with (b) SLM M turned off, (c) horizontal multiplication, (d) vertical multiplication, (e) diagonal multiplication.

bigger than a single SLM. For this purpose we combined the spatiotemporal multiplexing method with a $4 \mathrm{f}$ optical system. The main role of STM method was to simultaneously increase VFOV and remove gaps in a reconstructed image. The optical setup has been used in order to decrease image size to SLM M dimensions and increase it back to its original size afterwards. Application of $4 \mathrm{f}$ optical system brings some additional limitations put on a reconstructed image, however by proper choice of $4 \mathrm{f}$ system elements it is possible to reach significant increase of an image size for naked eye observation.

For synchronization of displayed holograms with modulation of SLM M we have developed a software based on GPU which provided the working frequency for four subframes equal to 12.5 frames/sec for SLM with theoretical frame refreshing rate $60 \mathrm{~Hz}$.

To present advantages of the developed display, we have shown the reconstructions of two objects: scaled rectangle and gargoyle. The first one allowed estimating how much an observer can see while different modulation frequencies are applied at the SLM M. The second one presented the viewing conditions for 3D object. Moreover, the presented results in an expressive way illustrate the "key hole" effect and viewing limitations connected with utilization of a single SLM.

In the described display we have succeeded in reconstruction and observation of an image in 4 times bigger VFOVy in comparison to a single SLM setup without STM method applied. Nevertheless, we believe that with further improvement of SLM's parameters our method can become even more efficient, providing viewer with display of higher SBP. Below we present the comparison of the display parameters which can be reached for $2 \mathrm{~K}$ ( $8 \mu \mathrm{m}$ pixel size) [23] and $4 \mathrm{~K}$ (3.74 $\mu \mathrm{m}$ pixel size) [27] SLM resolution. The comparison is made based on the assumption that $4 \mathrm{f}$ optical systems are optimized for these SLMs and provide angular magnification $M_{\alpha}=3$. Therefore, reconstructed image will be limited by SLM M aperture size only.

Table 1.Comparison of $2 \mathrm{~K}$ and $4 \mathrm{~K}$ SLMs observation parameters for the same $M_{\alpha}=3$.

\begin{tabular}{lcc}
\hline Horizontal definition & $2 \mathrm{~K}$ & $4 \mathrm{~K}$ \\
\hline Pixel size $\Delta_{\mathrm{x}}(\mu \mathrm{m})$ & 8 & 3.74 \\
Possible image dim. $\mathrm{W} \times \mathrm{H}(\mathrm{mm})$ & $46.08 \times 25.92$ & $45.52 \times 26.94$ \\
Min. reconstr. distance $z_{r}(\mathrm{~mm})$ & 700 & 320 \\
App. viewing angle $\alpha\left(^{\circ}\right)$ & 5.07 & 10.92 \\
\hline
\end{tabular}

Table 2. Comparison of $2 \mathrm{~K}$ and $4 \mathrm{~K}$ SLMs observation parameters for the same $z_{r}=700$.

\begin{tabular}{lcc}
\hline Horizontal definition & $2 \mathrm{~K}$ & $4 \mathrm{~K}$ \\
\hline Pixel size $\Delta_{\mathrm{x}}(\mu \mathrm{m})$ & 8 & 3.74 \\
4f angular magnification & $M_{\alpha}=3$ & $\mathrm{M}_{\alpha}=6$ \\
Reconstruction distance $\mathrm{z}_{\mathrm{r}}(\mathrm{mm})$ & 700 & 700 \\
FOV $(\mathrm{mm})$ & $46.55 \times 46.55$ & $99.57 \times 99.57$ \\
Possible image dim. $\mathrm{W} \times \mathrm{H}(\mathrm{mm})$ & $46.08 \times 25.92$ & $91.92 \times 53.88$ \\
App. viewing angle $\alpha\left(^{\circ}\right)$ & 5.07 & 9.43 \\
\hline
\end{tabular}


The presented values show that smaller pixel size gives bigger variety for possible setup configurations. For the same image size 4K SLMs provide an observer with nearly 2 times bigger viewing angle, since reconstruction distance is decreased. However, utilization of $4 \mathrm{~K}$ SLMs would be also beneficial if reconstruction distance remained the same, i.e. $z_{r}=700 \mathrm{~mm}$. The situation is presented in Table 2, where due to smaller pixel size of $4 \mathrm{~K}$ SLMs a bigger magnification of $4 \mathrm{f}$ setup can be implemented. From the comparison it can be seen that both the image size and the viewing angle increase in $4 \mathrm{~K}$ display configuration.

\section{Acknowledgements}

The authors acknowledge financial support from the National Science Centre, Poland, within the project realized in the program MAESTRO under agreement 2011/02/A/ST7/ 00365 and from Warsaw University of Technology within the statutory funds.

\section{References}

1. L. Onural, T. Sikora, J. Ostermann, A. Smolic, M.R. Civanlar, and J. Watson, "An assessment of 3DTV Technologies", NAB BEC Proceedings, (2006).

2. I. Sexton and Y. Surman, "Stereoscopic and autostereoscopic display systems", Signal Process. Mag.16, 85-99 (1999).

3. J. Hong, Y. Kim, H.J. Choi, J. Hahn, J.H. Park, H. Kim, S.W. Min, N. Chen, and B. Lee, "Three-dimensional display technologies of recent interest: principles, status, and issues", Invited. Appl. Opt., 50, No. 34 H87-H115 (2011).

4. T.-Ch. Poon, Digital Holography and Three-Dimensional Displays, Springer, Berlin 2006.

5. F. Yaras, H. Kang, and L. Onural, "State of the art. in holographic displays :a survey", J. Disp. Technol. 6, 443-454 (2010).

6. S. Reichelt, R. Haussler, G. Futterer, and N. Leister, "Depth cues in human visual perception and their realization in 3D displays", Proc. SPIE 7690, 76900B (2010).

7. SeeReal Technologies, http://www.seereal.com

8. M. Kujawinska, T. Kozacki, C. Falldorf, T. Meeser, B.M. Hennelly, P. Garbat, W. Zaperty, M. Niemelä, G. Finke, M. Kowiel, and T. Naughton, "Multiwavefront digital holographic television", Opt. Express 22, 2324-2336 (2014).

9. M. Stanley, R.W. Bannister, C.D. Cameron, S.D. Coomber, I.G. Cresswell, J.R. Hughes, V. Hui, P.O. Jackson, K.A. Milham, R.J. Miller, D.A. Payne, J. Quarrel, D.C. Scattergood, A.P. Smith, M.A.G. Smith, D.L. Tipton, P.J. Watson, P.J. Webber, and C.W. Slinger, "100-megapixel generated holographic images from active tiling: a dynamic and scalable electro-optic modulator system", Proc. SPIE, 5005, 247-258 (2003).

10. J. Khan, H. Kim, Y. Lim, G. Park, and B. Lee, "Wide viewing angle dynamic holographic stereogram with a curved array of spatial light modulator", Opt. Express 16, 12372-12386 (2008).
11. T. Kozacki, "On resolution and viewing of holographic image generated by 3D holographic display", Opt. Express 18, 27118-27129 (2010).

12. F. Yaras, H. Kang, and L. Onural, "Multi-SLM holographic display with planar configuration", Proc. of 3D TV Conference: The True CVision-Capture, Transmission and Display in 3D Video, Proc. IEEE, (2010).

13. T. Kozacki, M. Kujawińska, G. Finke, B. Hennelly, and N. Pandey, "Extended viewing angle holographic display system with tilted SLMs in a circular configuration", Appl. Optics, 51, 1771-1780 (2012).

14. F. Yaras, H. Kang, and L. Onural, "Circular holographic video display system”, Opt. Express 19, 9147-9156 (2011).

15. J. Hahn, H. Kim, Y. Lim, G. Park, and B. Lee, "Wide viewing angle dynamic holographic stereogram with a curved array of spatial light modulators", Opt. Express 16, 12372-12386 (2008).

16. A. Michałkiewicz, M. Kujawinska, T. Kozacki, X. Wang, and P.J. Bos, "Holographic three-dimensional displays with liquid crystal on silicon spatial light modulator", Proc. SPIE 5531, 85-94 (2004).

17. T. Kozacki, G. Finke, P. Garbat, W. Zaperty, and M. Kujawińska, "Wide angle holographic display system with spatiotemporal multiplexing", Opt. Express 20, 27473-27481 (2012).

18. G. Finke, M. Kujawińska, W. Zaperty, and T. Kozacki, "Spatiotemporal multiplexing method for big images observation in wide angle holographic display", 3DTV-Conference: The True Vision-Capture, Transmission and Display of 3D Video (3DTV-CON), Proc IEEE (2013).

19. Y. Liu, X. Pang, S. Jiang, and J. Dong, "Viewing-angle enlargement in holographic augmented reality using time division and spatial tiling", Opt. Express 21, 12068-12076 (2013).

20. Edmund Optics, www.edmundoptics.com

21. K. Yamamoto, Y. Ichihashi, T. Senoh, R. Oi, and T. Kurita, "3D objects enlargement technique using an optical system and multiple SLMs for electronic holography", Opt. Express 20, 21137-21144 (2012).

22. M.J. Bastiaans, "Wigner distribution function and its application to first-order optics", J. Opt. Soc. Am. 69, 1710-1716 (1980).

23. Holoeye, http://holoeye.com/spatial-light-modulators/discontinued-devices/

24. G. Finke, M. Kujawińska, and T. Kozacki, "Visual perception in multi SLM holographic displays", Appl. Opt. 54, 3560-3568 (2015).

25. R. Sitnik, M. Kujawińska, and J. Woźnicki, "Digital fringe projection system for large-volume 360-deg shape measurement", Opt. Eng. 41, 443-449 2002.

26. T. Kozacki, "Holographic display with tilted spatial light modulator", Appl. Opt. 50, 3579-3588 (2011).

27. Jasper Display, www.jasperdisplay.com 